\title{
Variable structure control for parabolic evolution equations
}

\author{
Laura Levaggi*
}

August 7, 2018

\begin{abstract}
In this paper it is considered a class of infinite-dimensional control systems in a variational setting. By using a Faedo-Galerkin method, a sequence of approximating finite dimensional controlled differential equations is defined. On each of these systems a variable structure control is applied to constrain the motion on a specified surface. Under some growth assumptions the convergence of these approximations to an ideal sliding state for the infinite-dimensional system is shown. Results are then applied to the Neumann boundary control of a parabolic evolution equation.
\end{abstract}

\section{Introduction}

Variable structure control methods and in particular sliding mode controls, are by now recognised as classical tools for the regulation of systems governed by ordinary differential equations in a finite dimensional setting. For an overview of the finite-dimensional theory see [15].

While being easy to design, they possess attractive properties of robustness and insensitivity with respect to disturbances and unmodelled dynamics. These characteristics are all the more important when dealing with infinite-dimensional systems. In many control applications such as heat transfer processes, chemical processes, flexible manipulators the state evolution is governed by a partial differential equation. The complexity of these plants results in models having significant degrees of uncertainty. Thus motivated, recent research has been devoted to the extension of sliding mode control and therefore the use of discontinuous feedback laws, to the infinite-dimensional setting. While earlier works 9, 10, 12 were confined to some special classes of systems, at present both theory and application of sliding mode control have been extended to a rather general setting [13, 11, 14, 4, 3, 5]. In particular in 13, the key concept of equivalent control is introduced in a general Hilbert space framework for evolution

${ }^{*}$ L. Levaggi is with Department of Mathematics, University of Genova, Via Dodecaneso 35 - 16146 Genova, Italy levaggi@dima.unige.it 
equations governed by unbounded linear operators that generate $C_{0}$-semigroups. Also it is shown that, under some stability assumptions, the ideal sliding can be uniformly approximated by "real" motions evolving in a boundary layer of the sliding manifold, thus ensuring the validity of the method for application purposes. The relationship between the equivalent control method and generalised solutions of infinite-dimensional systems with discontinuous right-hand side is presented in [4, 3].

All the results in the above cited literature only take into consideration distributed control systems, i.e. they deal with bounded input operators. In this paper we make a first attempt to consider the extension of sliding modes to a class of boundary control problems in a general setting. To the author's knowledge there exist only a few results in this direction in the linear case 1, 2, where by application of integral transformations the problem is reduced to the control of a finite-dimensional differential-difference equation. Our approach goes instead in the direction of [16. In Section 2 we define the general abstract variational framework in which we set up our control problem. In particular, the main assumptions we make on the operator governing the evolution, are weak continuity and coerciveness, so that both linear and non-linear operators are comprised in this setting. In Section 3 we present our main result: a Faedo-Galerkin method is used to construct a sequence of finite-dimensional approximations of the given problem. On each of these the standard variable structure control theory of [15] can be applied. We then assume that for each approximation a control law is chosen to constrain the evolution in a boundary layer of a given sliding manifold and study the limit as the dimensions diverge. We show that, under some growth assumption on the norm of these controls, a limit motion exists, which satisfies the sliding condition. Then, in Section 4 we apply the obtained results to the Neumann boundary control of a heat equation.

\section{Abstract setting and problem statement}

In this paper we are going to consider a class of parabolic partial differential equations with controllers acting on the boundary. In particular we will study the case of Neumann boundary conditions and finite dimensional control space. Also, we suppose that a manifold $S$ is given, on which we want to restrict the motion of our system. We then analyse the problem of the existence of an admissible control law for which this ideal sliding motion is possible.

Example 2.1 Before going into the details of the precise abstract setting of the problem, we show an example of application to give an idea of the family of systems we intend to study.

Let $\Omega$ be a bounded, open subset of $\mathbb{R}^{n}$ with smooth boundary $\Gamma, T>0$ and $\Delta$ be the laplacian differential operator on $\mathbb{R}^{n}$. Consider the following evolution 
equation

$$
\begin{array}{ll}
\frac{\partial Q}{\partial t}(t, x)=\Delta Q(t, x)+q(x) Q(t, x) & t \in(0, T), x \in \Omega \\
\frac{\partial Q}{\partial \nu}(t, \sigma)=u(t) g(\sigma) & t \in(0, T), \sigma \in \Gamma \\
Q(0, x)=Q_{0}(x) & x \in \Omega .
\end{array}
$$

Here $Q:[0, T] \times \Omega \rightarrow \mathbb{R}$ represents the evolution of the "state vector", $u:$ $[0, T] \rightarrow \mathbb{R}$ is a scalar control law, $g: \Gamma \rightarrow \mathbb{R}$ and $q: \mathbb{R}^{n} \rightarrow \mathbb{R}$ is bounded. This equation represents a model of heat conduction with both diffusion and heat generation (if $q$ is nonnegative). Now for $\gamma: \Omega \rightarrow \mathbb{R}$ we can define (informally) a sliding surface $S$ as the set of functions $f: \Omega \rightarrow \mathbb{R}$ such that

$$
\int_{\Omega} f(x) \gamma(x) d x=0
$$

In this case a sliding motion $Q(t, x)$ on $S$ would satisfy

$$
\int_{\Omega} Q(t, x) \gamma(x) d x=0, \quad t>0
$$

\subsection{Variational formulation}

The setting of the abstract problem follows [6, 7, 8]: let $V$ be a separable, reflexive Banach space, $H$ be a Hilbert space, $V \subset H$ with continuous injection. The space $H$ is identified with its dual, while we denote by $V^{\prime}$ the dual space of $V$, so that we have

$$
V \subset H \subset V^{\prime} .
$$

For $u_{1}, u_{2} \in H$ the scalar product in $H$ will be denoted by $\left(u_{1}, u_{2}\right)$ and the derived norm by $\left|u_{i}\right|$. We will denote by $\|\cdot\|$ the norm in $V$ and by $\|\cdot\|_{*}$ that in $V^{\prime}$. The dual pairing between the two spaces will be written as $\langle\cdot, \cdot\rangle$. Also, we will assume that on $V$ it is defined a semi-norm [.] such that

$$
[v]+\lambda|v| \geq \beta\|v\|, \quad \forall v \in V, \quad \text { for some } \lambda, \beta>0 .
$$

It is assumed that all the above (infinite-dimensional) spaces are real vector spaces; results can be extended to the complex case with the necessary modifications. For any $T>0$ we can define the following spaces of vector-valued functions:

$$
\begin{aligned}
& L^{2}(0, T ; V)=\left\{f:[0, T] \rightarrow V: \int_{0}^{T}\|f(t)\|^{2} d t<+\infty\right\} \\
& L^{\infty}(0, T ; H)=\left\{f:[0, T] \rightarrow H: \sup _{t \in[0, T]}|f(t)|<+\infty\right\} .
\end{aligned}
$$

The space $L^{2}\left(0, T ; V^{\prime}\right)$ can be defined analogously. Also, it is possible to define on these spaces a concept of derivative, in a distributional sense (see i. e. [7] Chapter III). The following result [8] will be useful in the sequel. 


\section{Theorem 2.1 Let}

$$
W(0, T)=\left\{f \in L^{2}(0, T ; V): \frac{d f}{d t} \in L^{2}\left(0, T ; V^{\prime}\right)\right\} .
$$

All functions in $W(0, T)$ are, after eventual modification on a null measure set, continuous from $[0, T]$ in $H$, i.e. $W(0, T) \subset C^{0}(0, T ; H)$.

For $t \in(0, T)$ let $A(t): V \rightarrow V^{\prime}$ be an operator satisfying the following assumptions:

- for all $v, w \in V$ the map

$$
t \mapsto\langle A(t) v, w\rangle \text { is measurable; }
$$

- for all $t$ and any $u, v, \omega \in V$ the map

$$
\alpha \mapsto\langle A(t)(u+\alpha v), w\rangle \text { is continuous; }
$$

- there exist constants $c_{1}>0, c_{2} \geq 0$ such that

$$
\|A(t) v\|_{*} \leq c_{1}\|v\|+c_{2}, \quad \forall v \in V
$$

- there exist constants $\alpha>0$ and $\nu \in \mathbb{R}$ such that

$$
\langle A(t) v, v\rangle \geq \alpha[v]^{2}+\nu|v|^{2} \forall v \in V .
$$

- $A(\cdot)$ is 2 -weakly continuous, i.e.

$$
\begin{aligned}
& v_{k} \rightarrow v \text { weakly in } W(0, T) \Longrightarrow \\
& \qquad A(\cdot) v_{k}(\cdot) \rightarrow A(\cdot) v(\cdot) \text { weakly in } L^{2}\left(0, T ; V^{\prime}\right) .
\end{aligned}
$$

Let $U \subset \mathbb{R}^{m}$ be closed and convex and let $f:[0, T] \times U \rightarrow V^{\prime}$ satisfy the following condition: there exists a constant $C>0$ such that for any $u:[0, T] \rightarrow$ $U, u \in L^{2}(0, T)$

$$
\int_{0}^{T}\|f(t, u(t))\|_{*}^{2} \leq C\|u\|_{2}^{2},
$$

where $\|u\|_{2}$ is the usual $L_{2}$-norm (it will always be understood that control laws $u$ take values in $U$, so that we will write $u \in L^{2}(0, T)$ instead of $L^{2}(0, T ; U)$ ).

We are now ready to write the abstract evolution equation we are going to study. The evolution of the system will be given by a vector-valued function $y \in W(0, T)$ satisfying the following abstract Cauchy problem

$$
\left\{\begin{array}{l}
\frac{d y}{d t}+A(t) y(t)=f(t, u(t)) \quad \text { q.o. } t \\
y(0)=y_{0}
\end{array}\right.
$$


with $u \in L^{2}(0, T)$ and for some $y_{0} \in H$ (by Theorem 2.1 this makes sense). The differential equation above as to be understood as an equality in the dual space $V^{\prime}$, i.e. setting

$$
a(t ; v, w)=\langle A(t) v, w\rangle, \quad t>0, v, w, \in V
$$

and in view of Theorem 2.1] the differential problem (9) is equivalent to the following variational formulation

$$
\left\{\begin{array}{l}
\frac{d}{d t}(y(t), v)+a(t ; y(t), v)=\langle f(t, u(t)), v\rangle \forall v \in V \\
y(0)=y_{0}
\end{array}\right.
$$

Existence and uniqueness results of the solution of such equations, under our assumptions, can be found in 6] under monotonicity assumptions and in [7, 8] for the linear case.

Example 2.2 Let us see how Example 2.1 fits into this framework. Let $H=$ $L^{2}(\Omega)$ and

$$
V=H^{1}(\Omega)=\left\{f \in H: \frac{\partial f}{\partial x_{i}} \in H i=1, \ldots, n\right\} .
$$

On $V$ we set $[v]^{2}=|\nabla v|^{2}$ and $\|v\|^{2}=[v]^{2}+|v|^{2}$. Let $v \in V$ be arbitrary; by scalar multiplication and using Green's formula one finds that the solution $Q$ of (1) has to satisfy

$$
\begin{aligned}
\frac{d}{d t}(Q(t, \cdot), v)= & \int_{\Omega} \Delta Q(t, x) v(x) d x+(q Q(t, \cdot), v) \\
= & -\int_{\Omega} \nabla_{x} Q(t, v) \cdot \nabla v(x) d x \\
& +\int_{\Gamma} u(t) g(\sigma) v(\sigma) d \sigma+(q Q(t, \cdot), v) .
\end{aligned}
$$

Therefore setting $y_{0}=Q_{0}$ and $y(t)=Q(t, \cdot)$ we get the (autonomous) variational formulation of our abstract setting in the form (11) with

$$
a(v, w)=(\nabla v, \nabla w)-(q v, w)
$$

and

$$
\langle f(t, u), v\rangle=\int_{\Gamma} u g(\sigma) v(\sigma) d \sigma
$$

Now (4) and (5) are easily verified and (6) follows from

$$
a(v, v)=[v]^{2}-(q v, v) \geq[v]^{2}-\left(\sup _{\Omega} q\right)|v|^{2} .
$$

Also, the operator $A$ defined as $\langle A v, w\rangle:=a(v, w)$ is linear and bounded, therefore it is 2-weakly continuous and we have (7).

Moreover, on $V$ the trace operator $\tau$ of restriction of a function to the boundary of $\Omega$ is well defined [8]. The range of $\tau$ is the Banach space $Z=H^{1 / 2}(\Gamma)$ and $\gamma$ is continuous from $V$ onto $H^{1 / 2}(\Gamma)$. Therefore $f$ is well defined for any $g$ in the dual of $H^{1 / 2}(\Gamma)$, hence for example for all $g \in L^{2}(\Gamma)$ and obviously satisfies (8) with $C=\|g\|_{L^{2}(\Gamma)}\|\tau\|_{\mathcal{L}(V, Z)}$. 


\section{Main results}

In this section we introduce the concept of sliding surface for the control problem (11) and show how sliding motions can be defined in this context.

Assume we are working in the framework set up in Section [2] Thanks to separability, there exists a countable basis for $V$, so that it is possible to define a family $\left\{V_{k}\right\}_{k \in \mathbb{N}}$ of finite dimensional subspaces of $V$

$$
V_{k}=\operatorname{span}\left\{v_{1, k}, \ldots, v_{N_{k}, k}\right\}
$$

such that

$$
V_{k} \subset V_{k+1}, \quad \bigcup_{k \in \mathbb{N}} V_{k}=V .
$$

Then it is possible to define approximate solutions of (11) by projecting on the subspaces $V_{k}$, using the standard Faedo-Galerkin method. We thus define the following family of variational problems: find $y_{k}:[0, T] \rightarrow V_{k}$ such that

$$
\left\{\begin{array}{l}
\frac{d}{d t}\left(y_{k}(t), v\right)+a\left(t ; y_{k}(t), v\right)=\left\langle f\left(t, u_{k}(t)\right), v\right\rangle \forall v \in V_{k} \\
y_{k}(0)=y_{0, k}
\end{array}\right.
$$

with $y_{0, k} \in V_{k}$ for all $k$ and a sequence $\left\{u_{k}\right\}$ in $L^{2}(0, T)$. Note that, since $V_{k}$ has dimension $N_{k}$, the above problem can be written as an ordinary differential equation. In fact, since $y_{k}(t) \in V_{k}$, there exists a vector $\xi_{k}(t) \in \mathbb{R}^{N_{k}}$ such that

$$
y_{k}(t)=\sum_{i=1}^{N_{K}}\left(\xi_{k}(t)\right)_{i} v_{i, k} .
$$

The differential equation in (14) is satisfied for all $v \in V_{k}$ iff it is valid for every element of the basis of $V_{k}$. Therefore, if

$$
\begin{gathered}
y_{0, k}=\sum_{i=1}^{N_{K}}\left(\xi_{0, k}\right)_{i} v_{i, k}, \\
f_{k}(t)=\left(\left\langle f\left(t, u_{k}(t)\right), v_{i, k}\right\rangle\right)_{i=1, \ldots, N_{k}},
\end{gathered}
$$

and

$$
\begin{array}{cl}
A_{k}(t)=\left(a_{i j}^{(k)}(t)\right)_{i, j=1, \ldots, N_{k}}, & a_{i j}^{k}(t)=a\left(t ; v_{i, k}, v_{j, k}\right), \\
M_{k}=\left(m_{i j}^{(k)}(t)\right)_{i, j=1, \ldots, N_{k}}, & m_{i j}^{k}(t)=\left(v_{i, k}, v_{j, k}\right),
\end{array}
$$

the differential problem (14) is equivalent to the following ordinary Cauchy problem

$$
\left\{\begin{array}{l}
M_{k} \dot{\xi}_{k}(t)+A_{k} \xi_{k}(t)=f_{k}(t) \\
\xi_{k}(0)=\xi_{0, k}
\end{array}\right.
$$

We now prove a convergence result for the approximations $y_{k}$, under some conditions on the controls sequence $\left\{u_{k}\right\}$. 
Theorem 3.1 Let the assumptions in Section Q be satisfied and $\left\{u_{k}\right\}$ be a sequence in $L^{2}(0, T)$. Let $y_{k}$ be the solution of (14) and suppose that $y_{0, k} \rightarrow y_{0}$ in $H$ for $k \rightarrow+\infty$. Suppose moreover that the following condition on the growth of the control norms is satisfied

$$
\left\|u_{k}\right\|_{L^{2}(0, t)}^{2} \leq M \int_{0}^{t}\left|y_{k}(s)\right|^{2} d s+N, t \leq T
$$

for some non-negative constants $M$ and $N$ and that $f$ is the following weak continuity assumption

$$
\begin{aligned}
& u_{k} \rightarrow u^{*} \text { weakly in } L^{2}(0, T) \text { then } \\
& \qquad f\left(\cdot, u_{k}(\cdot)\right) \rightarrow f(\cdot, u(\cdot)) \text { weakly in } L^{2}\left(0, T ; V^{\prime}\right)
\end{aligned}
$$

Then there exist a control law $u^{*} \in L^{2}(0, T)$ and a function $y^{*} \in W(0, T)$ verifying (11), such that, for some subsequence,

$$
\begin{aligned}
& y_{k} \rightarrow y^{*} \text { weakly in } W(0, T) \\
& y_{k} \rightarrow y^{*} \text { weakly* in } L^{\infty}(0, T ; H) \\
& u_{k} \rightarrow u^{*} \text { weakly in } L^{2}(0, T) .
\end{aligned}
$$

Proof. Writing (14) for $v=y_{k}(t)$ we get

$$
\left(\dot{y}_{k}(t), y_{k}(t)\right)+a\left(t ; y_{k}(t), y_{k}(t)\right)=\left\langle f\left(t, u_{k}(t)\right), y_{k}(t)\right\rangle \text {. }
$$

As the first term on the left is in fact the time derivative of $\left|y_{k}(t)\right|^{2} / 2$, integrating the above identity we have

$$
\begin{aligned}
\frac{1}{2}\left|y_{k}(t)\right|^{2}+\int_{0}^{t} a\left(t ; y_{k}(s), y_{k}(s)\right) d s= & \\
& \frac{1}{2}\left|y_{k}(0)\right|^{2}+\int_{0}^{t}\left\langle f\left(t, u_{k}(s)\right), y_{k}(s)\right\rangle d s .
\end{aligned}
$$

By (6), (8) and (2) we obtain the following inequality

$$
\begin{aligned}
\frac{1}{2}\left|y_{k}(t)\right|^{2}+\alpha \int_{0}^{t}\left[y_{k}(s)\right]^{2} d s & \\
\frac{1}{2}\left|y_{k}(0)\right|^{2}-\nu \int_{0}^{t}\left|y_{k}(s)\right|^{2} d s & \\
& +c\left\|u_{k}\right\|_{2}\left(\int_{0}^{t}\left[y_{k}(s)\right]^{2} d s+\int_{0}^{t}\left[y_{k}(s)\right]^{2} d s\right)^{1 / 2}
\end{aligned}
$$

for some constant $c>0$. Consider now for $x \geq 0$ the function $h(x)=(\alpha x) / 2-$ $c \sqrt{x}$. It is easy to show that it has minimum for $x=(c / \alpha)^{2}$, therefore $c \sqrt{x} \leq$ 
$\left(\alpha x+c^{2} / \alpha\right) / 2$, thus

$$
\begin{aligned}
& \frac{1}{2}\left|y_{k}(t)\right|^{2}+\frac{\alpha}{2} \int_{0}^{t}\left[y_{k}(s)\right]^{2} d s \leq \\
& \frac{1}{2}\left|y_{k}(0)\right|^{2}+\left(\frac{\alpha}{2}+|v|\right) \int_{0}^{t}\left|y_{k}(s)\right|^{2} d s+\frac{c^{2}}{2 \alpha}\left\|u_{k}\right\|_{2}^{2} .
\end{aligned}
$$

Now, since by hypothesis $\left|y_{0, k}-y_{0}\right|$ tends to zero, the term $\left|y_{k}(0)\right|^{2}$ is bounded. Moreover by (16)

$$
\left|y_{k}(t)\right|^{2}+\alpha \int_{0}^{t}\left[y_{k}(s)\right]^{2} d s \leq c_{1}+c_{2} \int_{0}^{t}\left|y_{k}(s)\right|^{2} d s
$$

for some constants $c_{1}, c_{2}>0$. Since $\alpha>0$ we get

$$
\left|y_{k}(t)\right|^{2} \leq c_{1}+c_{2} \int_{0}^{t}\left|y_{k}(s)\right|^{2} d s
$$

Therefore, by Gronwall's lemma we obtain for some constant $K>0$

$$
\left\|y_{k}\right\|_{L^{\infty}(0, T ; H)}=\sup _{t \in[0, T]}\left|y_{k}(t)\right| \leq K
$$

therefore from we also have

$$
\int_{0}^{T}\left[y_{k}(s)\right]^{2} d s \leq \mathrm{const}
$$

and lastly, using (2) and (5)

$$
\begin{aligned}
\left\|y_{k}\right\|_{L^{2}(0, T ; V)}= & \left(\int_{0}^{T}\left\|y_{k}(s)\right\|^{2} d s\right) \leq \mathrm{const}, \\
& \int_{0}^{T}\left\|A(t) y_{k}(t)\right\|_{*} d t \leq \mathrm{const} .
\end{aligned}
$$

Since spheres are weakly compact in both $L^{2}(0, T ; V)$ and $L^{2}\left(0, T ; V^{\prime}\right)$, weakly* compact in $L^{\infty}(0, T ; H)$, we can extract a subsequence of $\left\{y_{k}\right\}$ (which for simplicity we still denote by $\left.\left\{y_{k}\right\}\right)$ converging to some $y^{*} \in L^{2}(0, T ; V) \cap$ $L^{\infty}(0, T ; H)$ for both the weak topology of $L^{2}(0, T ; V)$ and the weak* topology of $L^{\infty}(0, T ; H)$ and such that $A y_{k}$ weakly converges to some $\eta$ in $L^{2}\left(0, T ; V^{\prime}\right)$. By (16) we also have that $\left\|u_{k}\right\|_{2}$ is bounded, thus eventually passing to a further subsequence, there exists $u^{*} \in L^{2}(0, T)$ such that $u_{k}$ converges to $u^{*}$ weakly in $L^{2}(0, T)$. Also, by (17) we can proceed as in the proof of Theorem 1.1, p. 159 of [6] to conclude that

$$
\left\{\begin{array}{l}
\frac{d}{d t} y^{*}(t)+\eta(t)=f\left(t, u^{*}(t)\right) \\
y(0)=y_{0}
\end{array}\right.
$$


Also, by a standard argument (see i.e. 16, Theorem 3) one can prove that $\dot{y}_{k} \rightarrow \dot{y}^{*}$ weakly in $L^{2}\left(0, T ; V^{\prime}\right)$, i.e. $y_{k} \rightarrow y^{*}$ weakly in $W(0, T)$. Thus, by (7) $\eta(t)=A(t) y^{*}(t)$ and the proof is complete.

Having achieved the above convergence result, we introduce as in [16], a set $D$ which can be either $V$ or a sufficiently large open subset of $H$ and a mapping $s: D \rightarrow \mathbb{R}^{m}$ continuously Fréchet differentiable on $D$. The sliding surface $S$ we consider is defined as $S=\{y \in D: s(y)=0\}$. Proceeding as in 16, by slightly modifying proofs, it is possible to prove the following

Corollary 3.1 Let the assumptions of Theorem 3.1 hold. Let $z_{k}(t)=s\left(y_{k}(t)\right)$ and assume that one of the following is satisfied:

(1) $D=V, s$ is affine and $z_{k} \rightarrow 0$ uniformly in $t$;

(2) $\mathcal{B}_{H}(0, K) \subset D, V$ is compactly embedded in $H$ (here $\mathcal{B}_{H}$ denotes a ball in $H$, while $K$ is defined in (19) above) and $z_{k}(t) \rightarrow 0$ for almost every $t \in[0, T]$.

Then the limit motion $y^{*}$ of Theorem 3.1 belongs to the sliding manifold $S$.

Remark 3.1 Note that by (15) every $y_{k}$ solves a finite-dimensional problem, thus for the approximate solutions all results of the classical theory of variable structure systems and sliding mode control of [15] are valid. Therefore existence results for system motions satisfying the requirements in Corollary [3.1] and design methods to achieve them are available. See also the discussion of existence under relaxed hypotheses developed in [16].

\section{An application}

In this Section we show an application of the obtained results on the control problem introduced in Example 2.1 We have already proved (see Example 2.2) that this partial differential equation with Neumann control fits in the abstract setting of Section 2 It is also easy to prove that for $f$ as in (13) the condition (17) is satisfied. In fact, if $u_{k} \rightarrow u$ weakly in $L^{2}(0, T)$, for any $\varphi \in L^{2}(0, T ; V)$ we have

$$
\begin{aligned}
& \int_{0}^{T}\left\langle\left[f\left(t, u_{k}(t)\right)-f(t, u(t))\right], \varphi(t)\right\rangle d t= \\
& \qquad \int_{0}^{T}\left[u_{k}(t)-u(t)\right] \int_{\Gamma} g(\sigma) \varphi(t)(\sigma) d \sigma d t
\end{aligned}
$$

which converges to zero since by Hölder's inequality and continuity of the trace operator on $V$

$$
\begin{aligned}
\int_{0}^{T}\left(\int_{\Gamma}|g(\sigma)||\varphi(t)(\sigma)| d \sigma\right)^{2} d t \leq & \\
& \|g\|_{L^{2}(\Gamma)}^{2} \int_{0}^{T}\|\varphi(t)\|^{2} d t<+\infty
\end{aligned}
$$


We then set $s: H \rightarrow \mathbb{R}, s(x)=(x, \gamma)$ and $S=\operatorname{ker} S$. For convenience we suppose that the chosen bases of the subspaces $V_{k}$ are orthonormal, so that the matrix $M_{k}$ in (15) is the identity (this is not restrictive since in the general case $M_{k}$ is symmetric, positive definite and a linear change of coordinates is sufficient to reconduct this problem to the orthonormal one). Then, setting $g_{k}=\left(\left(g, \tau v_{i, k}\right)_{L^{2}(\Gamma)}\right)_{i=1, \ldots, N_{k}}$, (15) can be rewritten as

$$
\left\{\begin{array}{l}
\dot{\xi}_{k}(t)+A_{k} \xi_{k}(t)=u_{k}(t) g_{k} \\
\xi_{k}(0)=\xi_{0, k}
\end{array}\right.
$$

Then $z_{k}(t)=s\left(y_{k}(t)\right)=\left(y_{k}(t), \gamma\right)=\gamma_{k}^{T} \xi_{k}(t)$, with $\gamma_{k}=\left(\left(v_{i, k}, \gamma\right)\right)_{i=1, \ldots, N_{k}}$. Let $V(t)=z_{k}^{2}(t) / 2$; then

$$
\dot{V}(t)=z_{k}(t) \dot{z}_{k}(t)=z_{k}(t)\left[\gamma_{k}^{T}\left(-A_{k} \xi_{k}(t)+u_{k}(t) g_{k}\right)\right] .
$$

By standard finite dimensional theory [15] a sliding mode exists on $S_{k}=\{x \in$ $\left.\mathbb{R}^{N_{k}}: \gamma_{k}^{T} x=0\right\}$ if $\gamma_{k}^{T} g_{k} \neq 0$. Also, in this case, setting

$$
u_{k}(t)=-U(t) \frac{\operatorname{sign}\left(z_{k}(t)\right)}{\gamma_{k}^{T} g_{k}}
$$

with $U(t)>\left|\gamma_{k}^{T} A_{k} \xi_{k}(t)\right|$ the sliding surface is globally attractive and reached in finite time. Moreover, if $\delta_{k}>0$ and $\left|s\left(y_{k}(0)\right)\right|<\delta_{k}$ the control

$$
u_{k}(t)=-\frac{U(t)}{\gamma_{k}^{T} g_{k}} \frac{z_{k}(t)}{\left|z_{k}(t)\right|+\delta_{k}}
$$

constrains the motion of the system in a $\delta_{k}$-boundary layer of $S_{k}$. Let us now consider the term $\gamma_{k}^{T} A_{k} \xi_{k}(t)$; since we assumed that the basis of $V_{k}$ is orthonormal, we have

$$
\gamma_{k}^{T} A_{k} \xi_{k}(t)=a\left(y_{k}(t), P_{k} \gamma\right)
$$

where $P_{k}: V \rightarrow V_{k}$ is the projection on $V_{k}$. Likewise we have

$$
\gamma_{k}^{T} g_{k}=\int_{\Gamma} g(\sigma)\left(P_{k} \gamma\right)(\sigma) d s .
$$

Thus, if for example $\gamma \in V$ and

$$
\int_{\Gamma} g(\sigma) \gamma(\sigma) d \sigma \neq 0
$$


since $P_{k} \gamma \rightarrow \gamma$ in $V$, there exists $K$ such that $\gamma_{k}^{T} g_{k} \neq 0$ for all $k \geq K$. In order to apply Theorem 3.1] we also have to show that (16) holds. Recalling that

$$
a\left(y_{k}(t), P_{k} \gamma\right)=\left(\nabla y_{k}(t), \nabla P_{k} \gamma\right)-\left(q y_{k}(t), P_{k} \gamma\right)
$$

we just have to show that, at least for suitable $\gamma$-s, the first term can be estimated using $\left|y_{k}(t)\right|$. Proceeding formally, by Green's formula we have

$$
\left(\nabla y_{k}(t), \nabla P_{k} \gamma\right)=-\left(y_{k}(t), \Delta\left(P_{k} \gamma\right)\right)
$$

$$
+\int_{\Gamma} y_{k}(t)(\sigma) \frac{\partial}{\partial \nu}\left(P_{k} \gamma\right)(\sigma) d \sigma .
$$

Thus (16) can be satisfied if sufficiently regular decompositions $\left\{V_{k}\right\}$ of $H^{1}(\Omega)$ are chosen and if the function $\gamma$ satisfies $\frac{\partial}{\partial \nu} P_{k} \gamma=0$, at least on some subsequence. For example this is true if $\gamma \in V_{N}$ for some $N$ and $\frac{\partial}{\partial \nu} \gamma=0$.

Remark 4.1 In this paper we have chosen a variational setting for our problem, by which we can encompass also some non-linear partial differential equations. For the linear case, another common abstract setting involves semigroup theory. In the above example our operator $A: V \rightarrow V^{\prime}$ could be, in some sense, substituted by $\mathcal{A}: \mathcal{D}(\mathcal{A}) \subset H \rightarrow H$, with

$$
\mathcal{D}(\mathcal{A})=\left\{y \in H^{2}(\Omega): \frac{\partial y}{\partial \nu}=0\right\}, \quad \mathcal{A} y=\Delta y+q y .
$$

Note that the last condition on $\gamma$ above is related to " $\gamma \in \mathcal{D}\left(\mathcal{A}^{*}\right)$ ", which is frequently encountered in the literature on output control of infinite-dimensional systems.

Remark 4.2 In many applications the function $z(t)$ of the example represents the system's output. The modulus of the control law we have chosen depends on the whole state norm, which could be unavailable for measurement. In [14] observers are designed to overcome this difficulty in the case of distributed control. It would be interesting to study their application to this case also.

\section{Conclusions and future work}

In this paper we have analysed the convergence behaviour of finite dimensional Faedo-Galerkin approximations of a class of variational problems, when sliding 
motions are taken into consideration. We have thus shown that, under some growth hypothesis on the norms of the controls, a sliding motion exists.

This is a first attempt to extend variable structure control to boundary control problems for infinite-dimensional systems and much work has still to be done in this area. Apart from the need to extend these results to different boundary control problems, it would be interesting to study how these results are related to a notion of equivalent control, which has already by introduced in

the infinite-dimensional setting and to approximability of ideal sliding motions by real ones.

\section{References}

[1] S. V. Drakunov and V. I. Utkin. Sliding mode control in dynamic systems. Internat. J. Control, 55(4):1029-1037, 1992.

[2] Sergey Drakunov and mit zgner. Generalized sliding modes for manifold control of distributed parameter systems. In Variable structure and Lyapunov control, Zinober, Alan S. I. (ed.), volume 193 of Lect. Notes Control Inf. Sci., pages 109-131. Springer-Verlag, Berlin, 1994.

[3] Laura Levaggi. Infinite dimensional systems' sliding motions. Eur. J. Control, 8(6):508-516, 2002.

[4] Laura Levaggi. Sliding modes in banach spaces. Differ. Integral Equ., 15(2):167-189, 2002.

[5] Laura Levaggi. High-gain feedback and sliding modes in infinite dimensional systems. Control Cybernet., 33(1):33-50, 2004.

[6] J.-L. Lions. Quelques méthodes de résolution des problèmes aux limites non linéaires. Dunod, Paris, 1969.

[7] J. L. Lions. Optimal Control of Systems Governed by Partial Differential Equations. Die Grundlehren der mathematischen Wissenschaften, Band 170. Springer-Verlag, New York, 1971.

[8] J.-L. Lions and E. Magenes. Non-homogeneous boundary value problems and applications. Vol. I. Die Grundlehren der mathematischen Wissenschaften, Band 181. Springer-Verlag, New York, 1972.

[9] Yu. V. Orlov and V. I. Utkin. Use of sliding modes in distributed system control problems. Automat. Remote Control, 43(9):1127-1135 (1983), 1982. 
[10] Yu. V. Orlov and V. I. Utkin. Sliding mode control in indefinite-dimensional systems. Automatica J. IFAC, 23(6):753-757, 1987.

[11] Yuri Orlov and Denis Dochain. Discontinuous feedback stabilization of minimum-phase semilinear infinite-dimensional systems with application to chemical tubular reactor. IEEE Trans. Automat. Control, 47(8):1293-1304, 2002 .

[12] Yuri Orlov and Vadim I. Utkin. Unit sliding mode control in infinitedimensional systems. Appl. Math. Comput. Sci., 8(1):7-20, 1998.

[13] Yuri V. Orlov. Discontinuous unit feedback control of uncertain infinitedimensional systems. IEEE Trans. Automat. Control, 45(5):834-843, 2000.

[14] Yury Orlov, Yiming Lou, and Panagiotis D. Christofides. Robust stabilization of infinite-dimensional systems using sliding-mode output feedback control. Internat. J. Control, 77(12):1115-1136, 2004.

[15] Vadim I. Utkin. Sliding modes in control and optimization. Communications and Control Engineering Series. Springer-Verlag, Berlin, 1992.

[16] Tullio Zolezzi. Variable structure control of semilinear evolution equations. In Partial differential equations and the calculus of variations, Vol. II, volume 2 of Progr. Nonlinear Differential Equations Appl., pages 997-1018. Birkhäuser, Boston, MA, 1989. 